\title{
Yersinia pestis insecticidal-like toxin complex (Tc) family proteins: characterization of expression, subcellular localization, and potential role in infection of the flea vector
}

\author{
Justin L Spinner ${ }^{1 *}$, Clayton O Jarrett ${ }^{1}$, Doris L LaRock², Samuel I Miller ${ }^{2,3}$, Carleen M Collins ${ }^{2^{\wedge}}$ \\ and B Joseph Hinnebusch ${ }^{1}$
}

\begin{abstract}
Background: Toxin complex (Tc) family proteins were first identified as insecticidal toxins in Photorhabdus luminescens and have since been found in a wide range of bacteria. The genome of Yersinia pestis, the causative agent of bubonic plague, contains a locus that encodes the Tc protein homologues YitA, YitB, YitC, and YipA and YipB. Previous microarray data indicate that the Tc genes are highly upregulated by $Y$. pestis while in the flea vector; however, their role in the infection of fleas and pathogenesis in the mammalian host is unclear.

Results: We show that the Tc proteins YitA and YipA are highly produced by Y. pestis while in the flea but not during growth in brain heart infusion (BHI) broth at the same temperature. Over-production of the LysR-type regulator YitR from an exogenous plasmid increased YitA and YipA synthesis in broth culture. The increase in production of YitA and YipA correlated with the yitR copy number and was temperature-dependent. Although highly synthesized in fleas, deletion of the Tc proteins did not alter survival of $Y$. pestis in the flea or prevent blockage of the proventriculus. Furthermore, YipA was found to undergo post-translational processing and YipA and YitA are localized to the outer membrane of $Y$. pestis. YitA was also detected by immunofluorescence microscopy on the surface of $Y$. pestis. Both YitA and YipA are produced maximally at low temperature but persist for several hours after transfer to $37^{\circ} \mathrm{C}$.

Conclusions: $Y$. pestis Tc proteins are highly expressed in the flea but are not essential for $Y$. pestis to stably infect or produce a transmissible infection in the flea. However, YitA and YipA localize to the outer membrane and YitA is exposed on the surface, indicating that at least YitA is present on the surface when $Y$. pestis is transmitted into the mammalian host from the flea.
\end{abstract}

Keywords: Yersinia pestis, Toxin complex proteins, YitA, YipA, YitR, Xenopsylla cheopis

\section{Background}

Yersinia pestis, the causative agent of bubonic plague, is maintained in nature by flea-rodent enzootic cycles and incidentally transmitted to humans through the bite of an infected flea. Like $Y$. pestis, the closely related Yersinia pseudotuberculosis and the more distantly related Yersinia enterocolitica harbor a virulence plasmid

\footnotetext{
* Correspondence: Justin. Spinner@nih.gov

Deceased

'Laboratory of Zoonotic Pathogens, Rocky Mountain Laboratories, NIAID, NIH, Hamilton, MT 59840, USA

Full list of author information is available at the end of the article
}

that encodes a type III secretion system (T3SS) and effector proteins (Yops). However, Y. pseudotuberculosis and $Y$. enterocolitica are not transmitted by fleas and cause enteric disease in humans [1-3]. Several $Y$. pestis genes have been found to be required to infect and be transmitted by fleas. These include the murine toxin gene $(y m t)$, the hemin storage (hmsHFRS) genes, the diguanylate cyclases encoded by $y 3730$ and $h m s T$, and $g m h A$. The $y 3730, h m s$, and $g m h A$ genes are needed for bis- $\left(3^{\prime}-5^{\prime}\right)$-cyclic dimeric GMP (c-di-GMP) metabolism, formation of an extracellular polysaccharide and a 


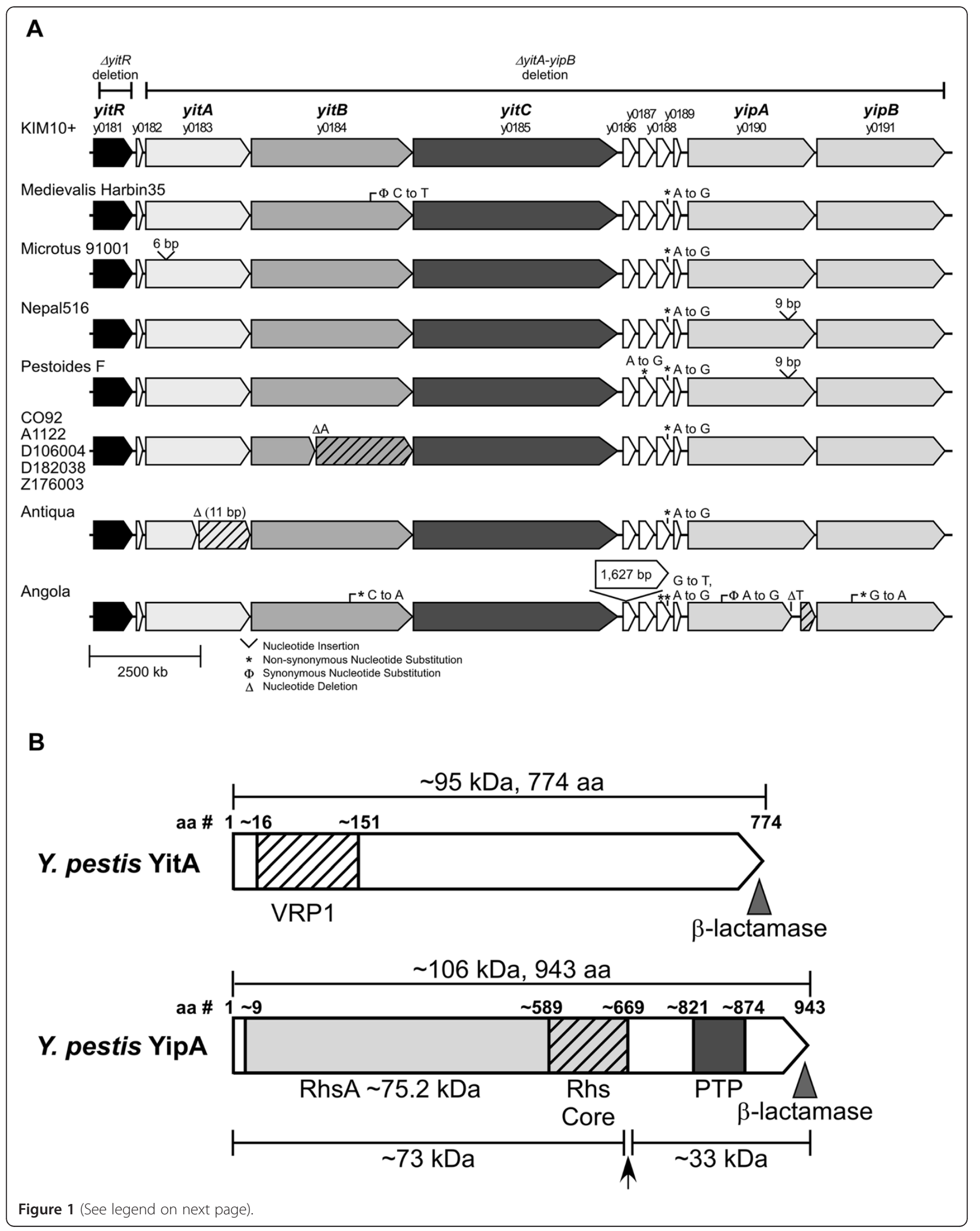


(See figure on previous page).

Figure $1 \mathrm{~A}$ ) The Tc protein locus of $Y$. pestis contains the yitABC and yipAB insecticidal-like protein genes and the upstream regulator yitR. Alignment of the Tc locus for all sequenced $Y$. pestis strains is shown with differences from KIM10+ indicated. The deletions in the $Y$. pestis KIM6+ $\Delta$ yitR and $\triangle$ yitA-yipB mutant strains used in this study are indicated. B) Domain structure of YitA and YipA. Hatch marks represent the region of YitA with similarity to the Salmonella virulence plasmid A (VRP1) protein family. The light gray area designates the region of YipA similar to the Rhs protein family. Light gray shaded hatch marks indicate the RHS repeat-associated core domain. Dark gray represents the region sharing homology to the protein tyrosine phosphatase (PTP) protein family and the PTP catalytic domain. The arrow indicates the inferred location of post-translational processing of YipA. The translational fusion junction of the full-length YitA and YipA with the mature $\beta$-lactamase is designated by shaded triangles.

lipopolysaccharide core modification, respectively, that are necessary for biofilm formation and blockage of the flea proventriculus [4-7]. The murine toxin $(y m t)$ gene, which encodes a phospholipase $\mathrm{D}$, is required for survival of $Y$. pestis within the flea midgut [8]. However, additional genes may also be important for survival and replication of $Y$. pestis within the flea or play a role in transmission to and survival within the mammalian host.

Recent microarray data indicate that a number of genes are differentially regulated by $Y$. pestis during infection of the flea compared to in vitro culture at the same temperature [9]. Among these were a group of upregulated genes that share homology with insect toxin genes of the Toxin complex (Tc) family. First identified in Photorhabdus luminescens, which maintains a symbiotic relationship with entomopathogenic nematodes of the family Heterorhabditidae $[10,11]$, Tc protein homologues are also found in a number of other bacteria including $Y$. enterocolitica and $Y$. pseudotuberculosis [12]. In P. luminescens, Tc genes are found at four loci which have a high degree of similarity and can be grouped into three basic genetic elements (tcdA/tcaAB/tccAB [type A], $t c d B / t c a C$ [type B], and $t c c C$ [type C]) [11]. The P. luminescens toxins are upregulated in the insect host [13], interact with each other to form large active toxin complexes and are highly insecticidal [14,15]. Furthermore, they have been shown to disrupt the actin cytoskeleton of NIH 3T3 Swiss mouse fibroblast cells $[15,16]$. More recently, P. luminescens toxin complexes were found to ADP-ribosylate actin and Rho GTPases, respectively, which caused actin polymerization and clustering in human HeLa cells and resulted in altered phagocytosis by Galleria mellonella hemocytes [17].

Tc protein homologues are found in all sequenced $Y$. pestis strains available to date (Figure $1 \mathrm{~A}$ ). Y. pestis $\mathrm{Tc}$ proteins are termed YitA (TcaA-like), YitB (TcaB-like), YitC (TcaC-like), and YipA and YipB (TccC-like) and are found within a single locus in the chromosome (Figure 1A) [18]. Although their sequences are highly conserved, $Y$. pestis strains CO92, A1122, D106004, D182038, and Z176003 have an apparent frameshift mutation in yitB (missing a single adenosine [A] from a string of seven A's), and strain Antiqua has an eleven nucleotide deletion resulting in a frameshift mutation in yitA. Additionally, $Y$. pestis Angola has a frameshift mutation in the C-terminus of yipA (Figure 1A). Previous studies have indicated that the Tc genes undergo thermoregulation, with greater expression after growth at $21^{\circ} \mathrm{C}$ or $26^{\circ} \mathrm{C}$ than $37^{\circ} \mathrm{C}$ [18-20]. Reverse transcription polymerase chain reaction data indicate that yit $A,-B,-C$ genes form an operon and $y i p A,-B$ genes are on a different transcriptional unit [18]. Deletion of the upstream LysR-like regulator (yitR) decreased the production of Tc proteins [18], indicating that YitR, which is also upregulated following growth of $Y$. pestis in the flea [9], is a positive regulator of expression. Similarly to $P$. luminescens, $Y$. pestis Tc proteins form a large multicomponent protein complex that contains all 5 Tc proteins [18]. Complex formation requires YitA and YitB, and YitC is necessary for association of YipA and YipB with the complex [18].

Although there is no defined biological role for the Yersinia Tc proteins, functional studies indicate that they are important in the interaction with insect cells or specific mammalian host cells. $Y$. pestis Tc proteins are not toxic to $M$. sexta [16], whereas $Y$. pseudotuberculosis and $Y$. enterocolitica (biotype 2-5, including strain W22703) Tc proteins are toxic, although they are much less potent than $P$. luminescens toxins $[12,21,22]$. Whereas $P$. luminescens toxins are also toxic to Xenopsylla cheopis rat fleas, $Y$. pestis and $Y$. pseudotuberculosis Tc proteins are not [2]. Additionally, $Y$. pseudotuberculosis and $Y$. pestis Tc proteins are not active against Spodoptera frugiperda (Sf9) insect cells [16]. However, unlike $Y$. pseudotuberculosis, $Y$. pestis Tc proteins are active against NIH 3T3 mouse fibroblast cells but not Caco-2 human intestinal epithelial cells [16], indicating specificity for certain host environments. There is evidence for T3SSdependent translocation of $Y$. pestis Tc proteins into host cells [18] and Tc genes (yit $A,-B,-C$ ) are upregulated within J774A.1 macrophages [23]. Furthermore, repression of Tc gene production $(-\Delta y i t R)$ increased phagocytosis by macrophages of $Y$. pestis isolated from fleas [9]. However, actual levels of the $Y$. pestis Tc proteins in the flea or during growth in liquid culture, or a potential role in survival within or transmission from the flea have not yet been determined.

In this study, we show that the Tc proteins YitA and YipA are highly produced by $Y$. pestis in the flea but not 
during growth in culture at the same temperature $\left(22^{\circ} \mathrm{C}\right)$ and that over-production of YitR increases YitA and YipA synthesis in vitro. YitA and YipA production was greatest during growth at lower temperatures (less than $22^{\circ} \mathrm{C}$ ) and minimally produced at $37^{\circ} \mathrm{C}$, although the proteins persisted for more than 9 hours after a transition from $22^{\circ} \mathrm{C}$ to $37^{\circ} \mathrm{C}$. YipA appears to be processed near the $\mathrm{C}$-terminus between the RhsA and PTP domains. Furthermore, YitA and YipA are localized to the outer membrane, and YitA is surface-exposed. We also show that the $Y$. pestis Tc proteins do not play a detectable role in $X$. cheopis infection or the ability to produce a transmissible infection.

\section{Results}

YitA and YipA are synthesized in the flea but not in vitro unless the YitR regulator is over-produced

A diagram of the $Y$. pestis Tc locus is shown in Figure 1a. $X$. cheopis fleas were infected with KIM6+ or KIM6+ $\triangle y i t A-y i p B$ (Figure 1A) to compare YitA and YipA (Figure 1B) protein levels following growth in the flea to growth in BHI culture. YitA and YipA were both highly produced by $Y$. pestis in the flea (Figure 2, lane 2) compared to stationary phase BHI cultures (Figure 2, lane 4) incubated at $22^{\circ} \mathrm{C}$, the same temperature at which the fleas were maintained. YitA was detected as a prominent band around $95 \mathrm{kDa}$, which corresponded to the expected size based on the YitA amino acid sequence. YipA was detected as two major bands. The smaller band at $\sim 73 \mathrm{kDa}$ was the most prominent. The larger band at $\sim 106 \mathrm{kDa}$ corresponds to the full length YipA predicted by its amino acid sequence and with recombinant YipA synthesized in and purified from E. coli (Figure 2, lane 9).

To determine if over-production of YitR would result in increased levels of YitA and YipA proteins during growth in vitro, the regulator yitR was cloned with its native promoter into the low-copy plasmid pWKS130 and the high-copy plasmid pCR-XL-TOPO. Y. pestis KIM6+ carrying pWKS130::yitR (Figure 2, lane 5) or pCR-XLTOPO::yitR (Figure 2, lane 6) had increased levels of YitA and YipA proteins following growth in $\mathrm{BHI}$ at $22^{\circ} \mathrm{C}$ compared to wild-type KIM6+ (Figure 2, lane 4) and KIM6+ $\Delta y i t A-y i p B$ (pCR-XL-TOPO::yitR) (Figure 2, lane 7). YitA and YipA protein increased with an increase in $y i t R$ copy number (Figure 2, lanes 5-6). The sizes of the YitA and YipB protein produced by all the strains under environmental conditions were similar (Figure 2, lanes 2, 5, 6). No detectable YitA or YipA protein was produced by the KIM6+DyitR deletion mutant (data not shown).

In vitro production of YitA and YipA by $Y$. pestis is dependent on growth temperature but not on culture medium

$Y$. pestis KIM6+, KIM6+ (pWKS130::yitR), and KIM6+ (pCR-XL-TOPO::yitR) were grown in $\mathrm{BHI}$ at $10^{\circ} \mathrm{C}, 22^{\circ} \mathrm{C}$, $28^{\circ} \mathrm{C}$, or $37^{\circ} \mathrm{C}$ overnight to determine YitA and YipA synthesis at different growth temperatures. YitA production in parental KIM6+ was detected after growth at $10^{\circ} \mathrm{C}$ (Figure $3 \mathrm{~A}$, lane 2). Full-size YipA was not detected in KIM6+ at any temperature (Figure 3A, lanes 2, 5, 8, and 11). When plasmid pWKS130::yitR was present, YitA was seen at all temperatures, with the maximum

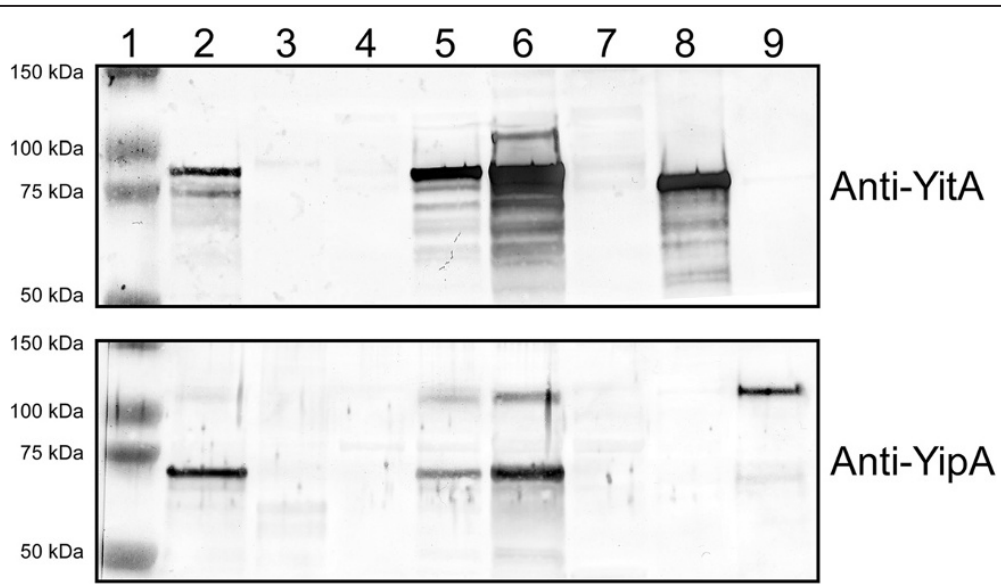

Anti-Ail

Figure 2 YitA and YipA are only detectable in Y. pestis isolated from fleas but over-production of YitR increases their synthesis in vitro. Lane 1, molecular weight ladder. Lane 2, Y. pestis KIM6+ isolated from infected fleas. Lane 3, KIM6+ KIM6+ grown at $22^{\circ} \mathrm{C}$ in BHI. Lane 5, KIM6+ (pWKS130:yitR) grown at $22^{\circ} \mathrm{C}$ in BHI. Lane 6, KIM6+ (pCR-XL-TOPO::yitR) grown at $22^{\circ} \mathrm{C}$ in BHI. Lane 7 , KIM6+ yitA-yipB (pCR-XL-TOPO::yitR) grown at $22^{\circ} \mathrm{C}$ in BHI. Lanes 8-9, recombinant YitA and YipA purified from E. coli. Panels show Western blots probed with anti-YitA, anti-YipA, or anti-Ail (sample loading control) antiserum. 


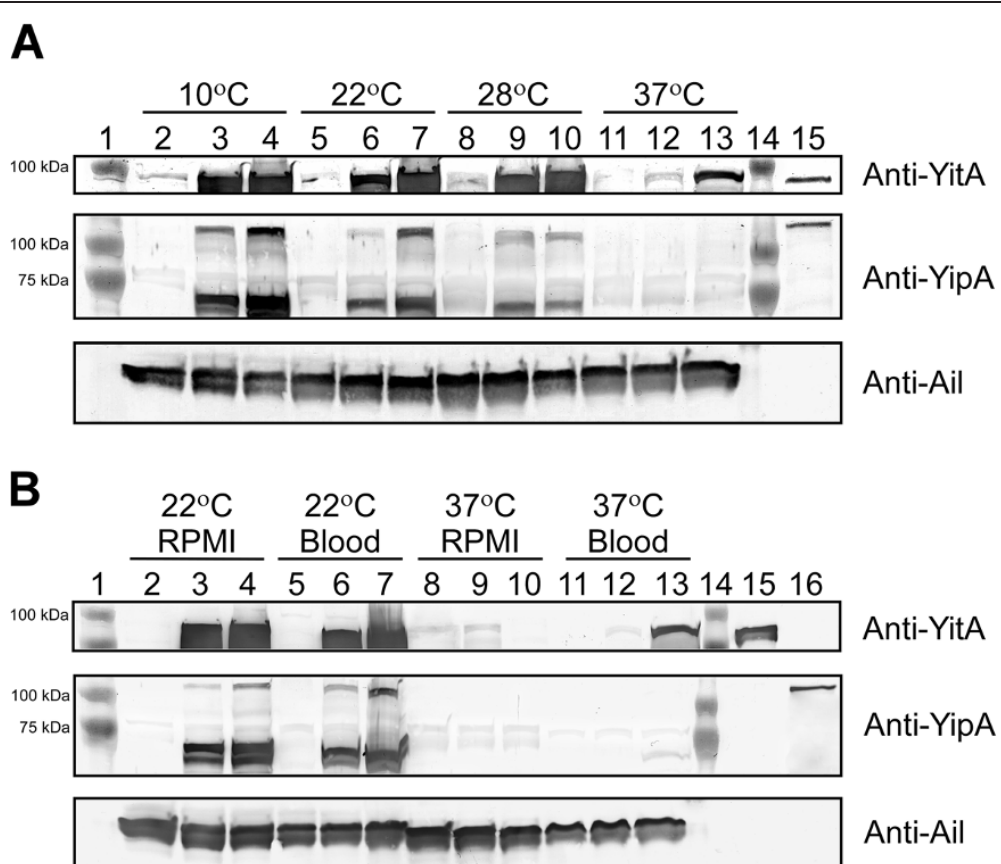

Figure 3 Maximal synthesis of YitA and YipA during growth at low temperatures. A) KIM6+ (lanes 2, 5, 8, and 11), KIM6+ (pWKS130::yitR) (lanes 3, 6, 9, and 12) and KIM6+ (pCR-XL-TOPO::yitR) (lanes 4, 7, 10, and 13) grown overnight at $10^{\circ} \mathrm{C}, 22^{\circ} \mathrm{C}, 28^{\circ} \mathrm{C}$ or $37^{\circ} \mathrm{C}$ in BHI broth. YitA and YipA purified from E. coli (lane 15). B) KIM6+ (lanes 2, 5, 8, and 11), KIM6+ (pWKS130:yitR) (lanes 3, 6, 9, and 12) and KIM6+ (pCR-XL-TOPO::yitR) (lanes $4,7,10$, and 13 ) grown overnight at $22^{\circ} \mathrm{C}$ or $37^{\circ} \mathrm{C}$ in either RPMI 1640 (RPMI) or whole sheep blood (Blood). YitA and YipA purified from E. coli (lanes 15 and 16). Panels show Western blots probed with anti-YitA, anti-YipA, or anti-Ail (sample loading control) antiserum.

level at $10^{\circ} \mathrm{C}$; the level decreased when the growth temperature was $37^{\circ} \mathrm{C}$ (Figure 3A, lanes 3, 6, 9, and 12). When plasmid pWKS130::yitR was present, YipA production was also greatest after growth at $10^{\circ} \mathrm{C}$ (Figure $3 \mathrm{~A}$, lane 3 ) and decreased when the growth temperature was $37^{\circ} \mathrm{C}$ (Figure 3A, lanes 6, 9, and 12); however, very little was seen at $37^{\circ} \mathrm{C}$ and the larger molecular weight band was no longer present (Figure 3A, lane 12). Y. pestis KIM6+ with the high-copy number pCR-XL-TOPO::yitR had the greatest production of YitA and YipA, which also decreased when the growth temperature was $37^{\circ} \mathrm{C}$ (Figure 3A, lanes 4, 7, 10, and 13). For each of the strains tested, levels of YitA and YipA were comparable after growth at $22^{\circ} \mathrm{C}$ or $28^{\circ} \mathrm{C}$ (Figure 3A, lanes 5 , 6, 7, 8, 9 and 10).

YitA and YipA production following growth in both blood and RPMI 1640 was equivalent to production following growth in BHI. YitA and YipA were produced to the greatest extent after growth at $22^{\circ} \mathrm{C}$ in $\mathrm{RPMI}$ 1640 and blood (Figure 3B, lanes 2-7) and levels dramatically decreased following growth at $37^{\circ} \mathrm{C}$ (Figure 3B, lanes 8-12). As with growth in BHI, $Y$. pestis with the high-copy pCR-XL-TOPO::yitR had the greatest level of production (Figure 3B, lanes 4, 7, 10, and 13) compared to KIM6+ with the low-copy YitR plasmid and wild-type KIM6+.
YitA and YipA persist for several hours following a growth temperature shift to $37^{\circ} \mathrm{C}$

YitA and YipA levels over time following a temperature shift from $22^{\circ} \mathrm{C}$ to $37^{\circ} \mathrm{C}$ was determined by Western blot analysis. YitA and YipA synthesized by KIM6+ (pCR-XLTOPO::yitR) following growth in $\mathrm{BHI}$ at $22^{\circ} \mathrm{C}$ were still present 7 hours after an upshift to $37^{\circ} \mathrm{C}$ (Figure 4, lanes 5, $8,11,14$, and 18). After 9 hours, a slight reduction in YitA and YipA protein was seen in the $37^{\circ} \mathrm{C}$ culture compared to the matched culture maintained at $22^{\circ} \mathrm{C}$ (Figure 4 , lanes 21 and 20, respectively). After 24 hours, there was a significant decrease in detectable YitA and YipA (Figure 4, lane 24) in the $37^{\circ} \mathrm{C}$ culture. After 30 hours at $37^{\circ} \mathrm{C}$, only a small quantity of YitA remained and no detectable YipA (Figure 4, lane 27).

\section{Evidence for post-translational processing of YipA}

Two forms of YipA were typically detected by Western blot: the predicted full-length protein at $\sim 106 \mathrm{kDa}$ and a smaller protein of $\sim 73 \mathrm{kDa}$, with the smaller form often predominating (Figures 2, 3, 4). To determine which of the bands detected using anti-YipA serum correspond to the $\mathrm{N}$-terminal region and the $\mathrm{C}$-terminal region of YipA, $Y$. pestis strains containing translational fusions of mature $\beta$-lactamase $(\sim 28.9 \mathrm{kDa})$ to the $\mathrm{C}$-terminus of YitA or YipA were constructed (Figure 1B). After overnight 

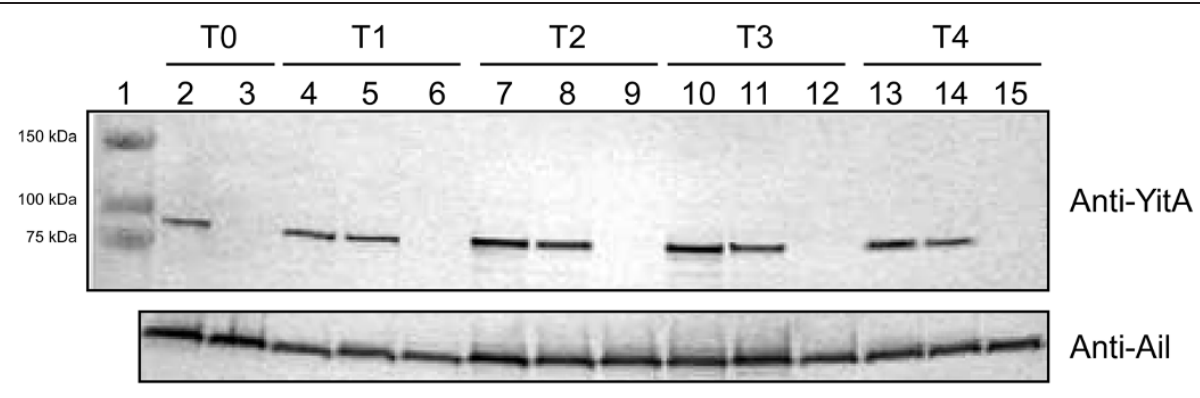

Anti-Ail

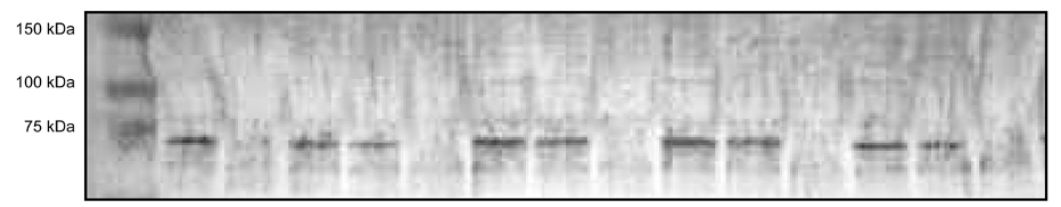

Anti-YipA
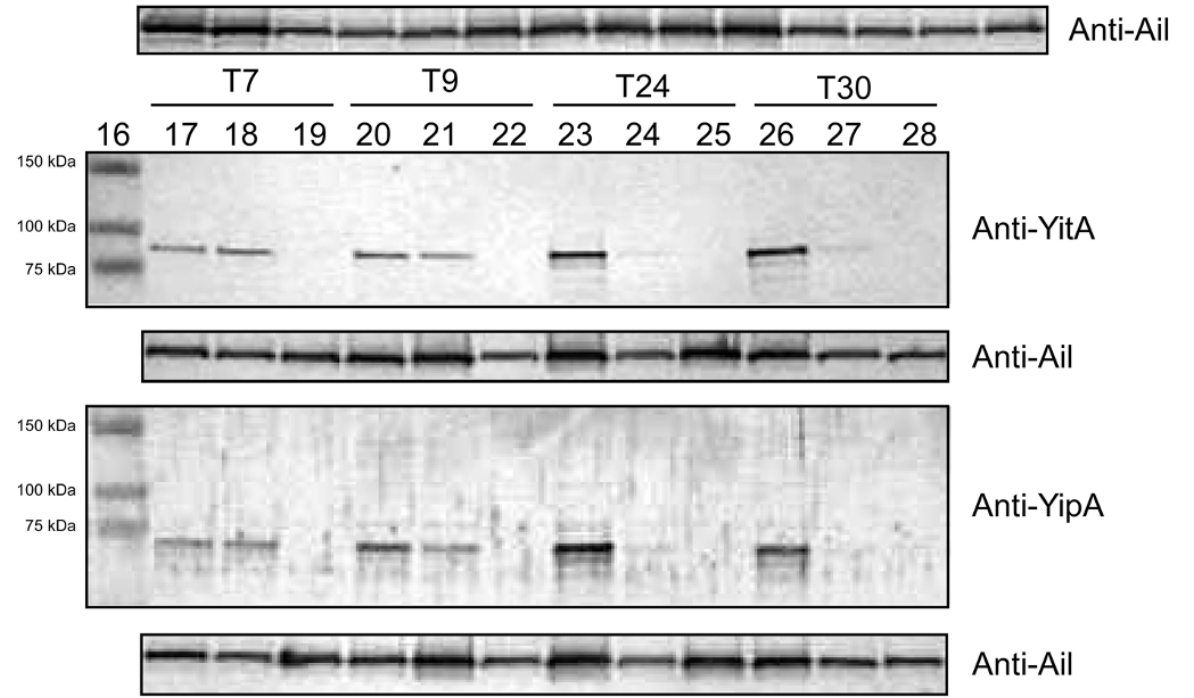

Figure 4 YitA and YipA proteins persist in $Y$. pestis for at least 9 hours after transfer to $37^{\circ} \mathrm{C}$. Y. pestis KIM6+ (pCR-XL-TOPO::yitR) (lanes 5 , $8,11,14,18,21,24$, and 27) and KIM6+ $\Delta$ yitA-yipB (pCR-XL-TOPO::yitR) (lanes 3, 6, 9, 12, 15, 19, 22, 25, and 28) were grown overnight at $22^{\circ} \mathrm{C}$ and subsequently transferred to $37^{\circ} \mathrm{C}$ for the indicated amount of time prior to sample collection. A matched KIM6+ (pCR-XL-TOPO::yitR) was maintained at $22^{\circ} \mathrm{C}$ as a positive reference control (lanes $2,4,7,10,13,17,20,23$, and 26 ). $T 0=$ initial time point, $T(x)=x$ hours at $37^{\circ} \mathrm{C}$.

Panels show Western blots probed with anti-YitA, anti-YipA, or anti-Ail (sample loading control) antiserum.

growth at $22^{\circ} \mathrm{C}, Y$. pestis YitA- $\beta$-lactamase and YipA- $\beta$ lactamase with or without plasmid pCR-XL-TOPO::yitR were assayed by Western blot. YitA- $\beta$-lactamase was detected by anti-YitA serum as a single band at $\sim 123 \mathrm{kDa}$ (due to the addition of the mature $\beta$-lactamase) with a light smear of smaller bands (Figure 5A, lane 2), whereas wild-type YitA was detected around $95 \mathrm{kDa}$ (Figure 5A, lane 4). Anti- $\beta$-lactamase antibody also detected full length YitA- $\beta$-lactamase at $\sim 123 \mathrm{kDa}$ as a prominent band and a smear of several smaller bands (Figure 5C, lane 2).

Anti-YipA serum detected YipA- $\beta$-lactamase as two prominent bands. The YipA- $\beta$-lactamase lower band at $\sim 73 \mathrm{kDa}$ (Figure 5B, lane 4) was the same size as the lower band seen with wild-type YipA (Figure 5B, lane 2). The upper band of YipA- $\beta$-lactamase was detected at $~ 135 \mathrm{kDa}$ (Figure 5B, lane 4), whereas the upper band of wild-type YipA was detected at $\sim 106 \mathrm{kDa}$ (Figure $5 \mathrm{~B}$, lane 2 ). Anti- $\beta$ lactamase antibody detected the upper $\sim 135 \mathrm{kDa}$ band corresponding to full-length YipA- $\beta$-lactamase (Figure $5 \mathrm{C}$, lane 4). However, the lower $\sim 73 \mathrm{kDa}$ band was not detected by anti- $\beta$-lactamase antibody (Figure $5 \mathrm{C}$, lane 4 ); although a distinct band at $\sim 62 \mathrm{kDa}$ was detected by anti$\beta$-lactamase antibody (Figure 5C, lane 4). This indicates that the YipA molecular weight band detected by antiYipA at $\sim 73 \mathrm{kDa}$ (Figure $5 \mathrm{~B}$, lane 4 ) represents the $\mathrm{N}$-terminus of YipA, whereas the smaller molecular weight band detected by anti- $\beta$-lactamase antibody $(\sim 62 \mathrm{kDa})$ represents the $\mathrm{C}$-terminal region of YipA fused to $\beta$-lactamase (Figure $5 \mathrm{C}$, lane 4 ). 


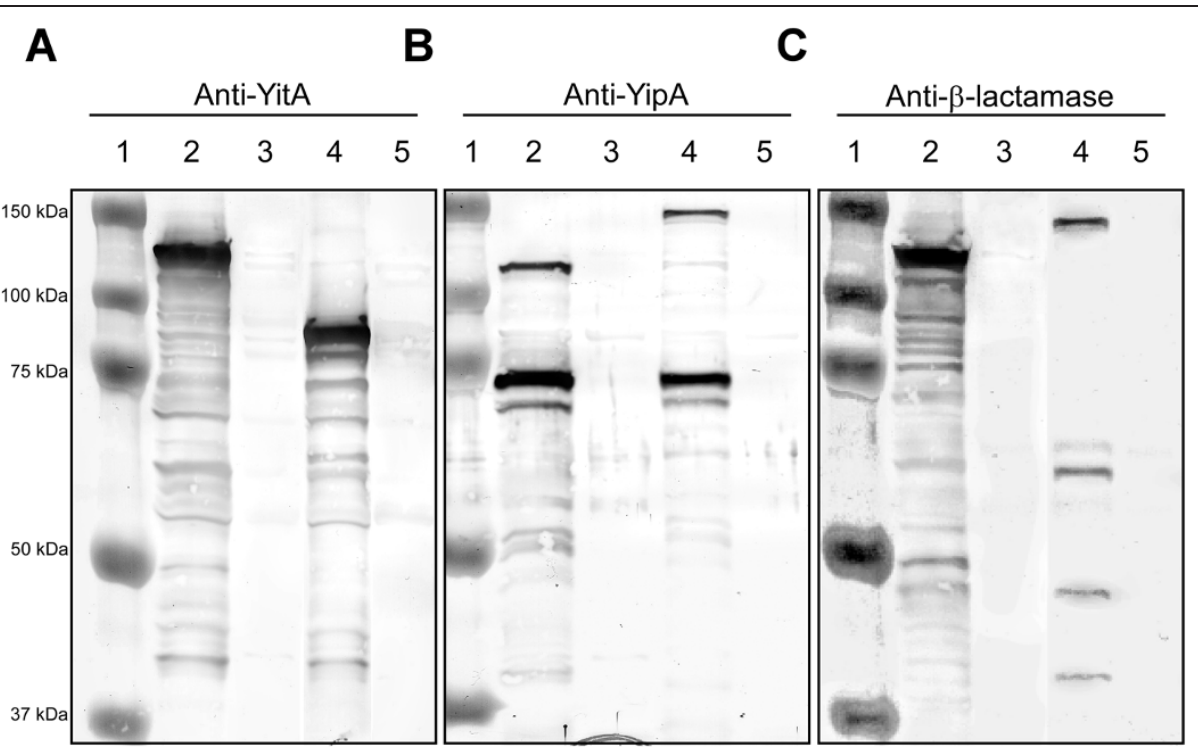

Figure $\mathbf{5}$ Characterization of post-translational processing of YipA. KIM6+ (pCR-XL-TOPO::yitR) with the C-terminus of YitA (Lane 2) or YipA (Lane 4) tagged with mature $\beta$-lactamase were grown overnight at $22^{\circ} \mathrm{C}$ in BHI broth. KIM6+ YitA- $\beta$-lactamase (Lane 3) or YipA- $\beta$-lactamase (Lane 5) not over-producing YitR grown overnight at $22^{\circ} \mathrm{C}$ in $\mathrm{BH}$ broth are provided as negative controls. Panels show Western blots probed with A) anti-YitA, B) anti-YipA, or C) anti- $\beta$-lactamase antiserum.

\section{YitA and YipA are localized in the outer membrane of $Y$. pestis}

To determine where YitA and YipA are localized within $Y$. pestis, cytoplasmic, periplasmic, inner membrane and outer membrane fractions were collected from KIM6+ YitA- $\beta$-lactamase (pCR-XL-TOPO::yitR) and KIM6+ YipA$\beta$-lactamase (pCR-XL-TOPO::yitR) grown in $\mathrm{BHI}$ overnight at $22^{\circ} \mathrm{C}$. YitA- $\beta$-lactamase was detected by anti-YitA (Figure 6a, top panel) and anti- $\beta$-lactamase (Figure 6A, bottom panel) antibodies predominately in the outer membrane fraction (Figure 6A, lane 6) and to a lesser extent in the periplasm (Figure 6A, lane 4). Wild-type YitA was detected in the cytoplasmic, periplasmic, inner membrane and outer membrane fractions of KIM6+ YipA- $\beta$ lactamase (Figure 6A, lanes 8-11).

Full length YipA- $\beta$-lactamase was detected by antiYipA (Figure 6A, middle panel) and anti- $\beta$-lactamase antibodies predominately in the periplasm and outer membrane fractions (Figure 6A, lanes 9 and 11) whereas the smaller $(\sim 73 \mathrm{kDa})$ YipA band was only detected by anti-YipA serum and was present in all of the fractions at approximately the same concentration (Figure 6A, lanes 8-11). Similarly, full-length wild-type YipA was detected by anti-YipA serum primarily in the periplasm and outer membrane fractions (Figure 6A, lanes 4 and 6), with the smaller $(\sim 73 \mathrm{kDa})$ band present in all the fractions of KIM6+ YitA- $\beta$-lactamase (Figure 6A, lanes 3-6). Interestingly, the smaller $(\sim 62 \mathrm{kDa})$ YipA $\beta$-lactamase band detected by anti- $\beta$-lactamase antibodies was predominately in the periplasm and inner membrane fractions (Figure 6A, lanes 9 and 10) and only minimally present in the cytoplasm and outer membrane fractions of KIM6+ YipA- $\beta$-lactamase (Figure 6A, lanes 8 and 11). Ail, a known outer membrane protein, was used as a loading and fractionation validation control and, as expected, was detected predominately in the outer membrane fractions of both bacterial strains. Thus, although YitA and YipA were detected in all of the fractions, the full length proteins are predominately localized within the periplasm and the outer membrane fractions. Conversely, the $\mathrm{N}$-terminus of processed YipA $(\sim 73 \mathrm{kDa})$ appears equally in all fractions and some quantity of the $\mathrm{C}$-terminal region of YipA- $\beta$-lactamase $(\sim 62 \mathrm{kDa})$ may be retained within the inner membrane fraction.

Immunofluorescence microscopy detected YitA on the surface of paraformaldehyde fixed KIM6+ (pCR-XLTOPO::yitR) (pAcGFP1) (Figure 6B, top row) but not on the surface of KIM6+ $y$ itA-yipB (pCR-XL-TOPO::yitR) (pAcGFP1) (Figure 6B, bottom row). YipA could not be detected above background levels on the surface of KIM6+ (pCR-XL-TOPO::yitR) (pAcGFP1) using anti-YipA serum (data not shown).

\section{Evaluation of the role of Tc proteins during $Y$. pestis flea infection}

To determine if the $Y$. pestis Tc proteins are important for survival within the flea or are required to produce a transmissible infection, we infected $X$. cheopis fleas with

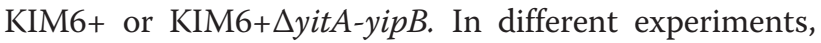
fleas were fed on blood containing a low infectious dose $\left(\sim 1 \times 10^{7} \mathrm{CFU}\right)$ or a high infectious dose $\left(\sim 1 \times 10^{8} \mathrm{CFU}\right)$

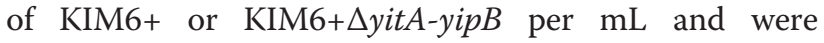



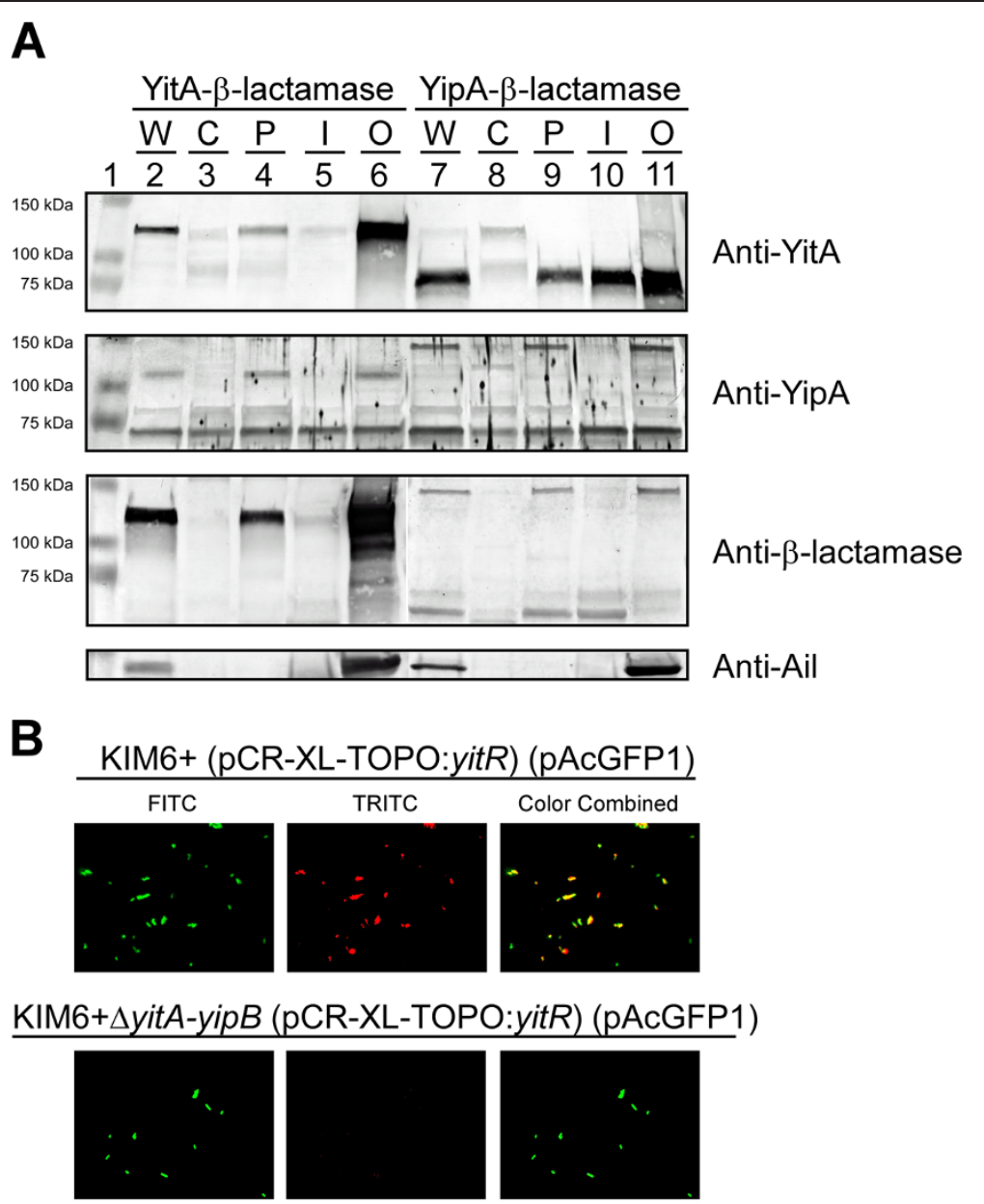

Figure 6 YitA and YipA are localized to the outer membrane fraction of $Y$. pestis and YitA is detectable on the surface of the bacteria. A) Y. pestis KIM6+ (pCR-XL-TOPO::yitR) YitA- $\beta$-lactamase (Lanes 2-6) or YipA- $\beta$-lactamase (Lanes 7-11) grown overnight at $22^{\circ} \mathrm{C}$ in $\mathrm{BHI}$ were lysed and separated into cytoplasmic (C), periplasmic (P), cytosolic inner membrane (I), and outer membrane (O) fractions and analyzed by Western blot. Whole cell lysates $(\mathrm{W})$ are provided as a control for both strains. Panels show Western blots probed with antisera to YitA, YipA, and $\beta$-lactamase, or Ail (a known Y. pestis outer membrane protein). B) Evidence of surface exposed YitA on Y. pestis. The top panel includes images of $Y$. pestis KIM6+ (pCR-XL-TOPO::yitR) (pAcGFP1, fluoresces green) grown overnight at $22^{\circ} \mathrm{C}$ in $\mathrm{BHI}$. YitA was detected by incubating fixed bacteria with anti-YitA serum and staining with Alexa Fluor 568 goat anti-rabbit lgG (fluoresces red). Fluorescence was imaged under green (FITC) and red (TRITC) filters, artificially colored, and merged. Images of KIM6+ $\triangle$ yitA-yipB (pCR-XL-TOPO::yitR) (pAcGFP1) prepared and imaged by the same method are shown in the bottom row as a background control.

maintained for 4 weeks. As expected, infection rates and the incidence of proventricular blockage increased with the number of bacteria in the infectious blood meal, but there were no differences in these rates between fleas infected with KIM6+ or with KIM6+ $y$ it A-yipB (Table 1). The average bacterial load per infected flea was also similar for the two strains. Thus, although highly produced in the flea gut, the $Y$. pestis Tc proteins do not appear to be necessary to establish a normal transmissible infection in the insect vector.

\section{Discussion}

In this study, we show that YitA and YipA proteins are highly produced by $Y$. pestis isolated from the flea vector
$X$. cheopis but not by $Y$. pestis grown in vitro unless the positive regulator $y i t R$ is over-expressed (Figure 2). This is consistent with microarray data showing a 6-50 fold increase in Tc gene expression in the flea, compared to $Y$. pestis grown in culture at the same temperature $[2,9]$. Previous data showed that deletion of yitR reduced Tc protein synthesis [18]. Additionally, expression of $y i t R$ is also upregulated in the flea [9]. Thus, we added $y i t R$ to $Y$. pestis on a low-copy and a high-copy plasmid, and found that the greatest levels of YitA and YipA were seen when $y i t R$ was present on the high-copy number plasmid (Figure 2). Furthermore, consistent with previous quantitative real-time polymerase chain reaction results [9], we found that deletion of yitR dramatically 
Table 1 Flea infection results with KIM6+ and KIM6+

\begin{tabular}{|c|c|c|c|c|c|c|c|c|}
\hline \multirow[t]{2}{*}{ Strain } & \multirow{2}{*}{$\begin{array}{l}\text { CFU/mL in } \\
\text { blood meal }\end{array}$} & \multicolumn{3}{|c|}{ CFU/infected flea ${ }^{a}$} & \multicolumn{3}{|c|}{$\%$ Fleas infected ${ }^{b}$} & \multirow{2}{*}{$\begin{array}{l}\% \text { Fleas } \\
\text { blocked }\end{array}$} \\
\hline & & Day 0 & Day 7 & Day 28 & Day 0 & Day 7 & Day 28 & \\
\hline KIM6+ & $1.04 \mathrm{e} 7$ & $3.91 \mathrm{e} 3 \pm 6.45 \mathrm{e} 2$ & $1.84 \mathrm{e} 5 \pm 3.51 \mathrm{e} 4$ & $3.79 \mathrm{e} 5 \pm 4.82 \mathrm{e} 4$ & 100.0 & 85.0 & 85.0 & 29.0 \\
\hline KIM6+ $\Delta$ yitA-yip B & $1.75 \mathrm{e} 7$ & $5.95 \mathrm{e} 3 \pm 1.03 \mathrm{e} 3$ & $2.61 \mathrm{e} 5 \pm 6.40 \mathrm{e} 4$ & $4.24 \mathrm{e} 5 \pm 6.86 \mathrm{e} 4$ & 100.0 & 75.0 & 80.0 & 33.0 \\
\hline KIM6+ & $5.20 \mathrm{e} 7$ & $1.66 \mathrm{e} 4 \pm 2.00 \mathrm{e} 3$ & $6.16 \mathrm{e} 5 \pm 1.21 \mathrm{e} 5$ & $4.99 \mathrm{e} 5 \pm 1.00 \mathrm{e} 5$ & 100.0 & 95.0 & 80.0 & 49.0 \\
\hline KIM6+DyitA-yip B & $1.55 e 8$ & $4.16 \mathrm{e} 4 \pm 3.82 \mathrm{e} 3$ & $5.30 \mathrm{e} 5 \pm 1.12 \mathrm{e} 5$ & $4.75 e 5 \pm 1.13 e 5$ & 100.0 & 80.0 & 75.0 & 51.0 \\
\hline
\end{tabular}

a Mean \pm standard error of CFU counts from 20 individual female fleas collected on the indicated day after infection.

$b$ Percentage of 20 female fleas collected on the indicated day after infection from which $Y$. pestis CFU were recovered.

$c$ Percentage of fleas that became blocked during the 28 days after infection.

reduced YitA and YipA levels after growth in the flea (data not shown). This validates the premise that YitR acts as a positive regulator of yitA and yipA expression in vivo. Since YitA and YipA were not detected in culture-grown $Y$. pestis KIM6+ and collection of sufficient bacteria from fleas for multiple experiments is not feasible, the use of YitR over-producing strains were used judiciously to further study YitA and YipA.

$Y$. pseudotuberculosis Tc proteins were preferentially produced after growth at $28-37^{\circ} \mathrm{C}$ but not at $15^{\circ} \mathrm{C}$ [16]. $Y$. pestis Tc proteins have also been shown to be produced after growth at $30^{\circ} \mathrm{C}$ [18]. However, microarray data indicate that $Y$. pestis Tc yit genes are preferentially transcribed at $21^{\circ} \mathrm{C}$ or $26^{\circ} \mathrm{C}$ and down-regulated (3-fold for $y i t A$ and 4.2 -fold for $y i t R)$ after growth at $37^{\circ} \mathrm{C}[19,20]$. This thermoregulation is also seen with $Y$. enterocolitica W22703 Tc genes, which show a preference for lowtemperature expression and have markedly downregulated expression at $37^{\circ} \mathrm{C}$ [22]. Correspondingly, our data show that YitA and YipA are both thermoregulated in KIM6+, with the greatest production during growth at lower temperatures and only minimal production during growth at $37^{\circ} \mathrm{C}$ (Figure 3A). Furthermore, YitA and YipA underwent similar thermoregulation after growth in both RPMI 1640 and blood (Figure 3B.). Thus, YitA and YipA would not be expected to play a role in $Y$. pestis pathogenesis late in the course of mammalian infection. This is supported by gene expression data from $Y$. pestis isolated from rat bubos that show no detectable expression of $y i t R$, and 2-25 fold less expression of yitA, $B, C$ and yipB than $Y$. pestis isolated from fleas $[9,20,24]$. However, yit $A,-B,-C$ were all found to be upregulated 1.3- to 7.6-fold by $Y$. pestis within J774A.1 macrophage-like cells compared to bacteria grown in cell culture medium under the same conditions [23], indicating that the optimum environment for Tc protein production at $37^{\circ} \mathrm{C}$ may be within host phagocytes.

Western blot analysis of YitA and YipA proteins from $Y$. pestis reveals potential processing of YipA (Figure 2 and 3). YipA was consistently detected by anti-YipA serum as two distinct protein bands of $\sim 106 \mathrm{kDa}$ and $\sim 73 \mathrm{kDa}$ (Figure 2). From the amino acid sequence,
YipA is predicted to be $\sim 106 \mathrm{kDa}$. Thus, YipA may be present as a full-length protein and a processed variant. We show that an anti- $\beta$-lactamase antibody only detected the $\sim 135-\mathrm{kDa}$ full-length YipA- $\beta$-lactamase protein but not the lower weight band expected at $\sim 102 \mathrm{kDa}$ $(73 \mathrm{kDa}+29 \mathrm{kDa})$ (Figure 5). This indicates that the $73-\mathrm{kDa}$ band detected with anti-YipA serum is the $\mathrm{N}$-terminus of the processed YipA. In support of this, the anti- $\beta$-lactamase antibody also detected a prominent smaller band which migrated a little over half the distance between 50 and $75 \mathrm{kDa}$ at $\sim 62 \mathrm{kDa}$. This band would correspond with the cleaved C-terminus of YipA $(\sim 33 \mathrm{kDa})$ bound to $\beta$-lactamase $(29 \mathrm{kDa})$. Although both YipA bands were consistently seen in repeat experiments, there were smaller variable bands and smearing often seen using anti-YipA antibody and anti- $\beta$-lactamase antibodies. This suggests that the processed YipA is not stable and may undergo degradation under our assay conditions. The processed state of these proteins under natural conditions is difficult to explore due to limitations in the collection of bacteria from fleas. Nonetheless, the $\mathrm{N}$ and C-terminal regions of YitA and YipA contain predicted domains (Figure 1B). The N-terminus of YitA contains a domain that shares similarity with the Salmonella virulence plasmid A (VRP1) protein family. The YipA amino acid sequence indicates two conserved domains, including an $\mathrm{N}$-terminus that shares similarity with the Rhs protein family reported in cell envelope biogenesis and outer membrane proteins. The YipA RhsA domain is predicted to be approximately $75.4 \mathrm{kDa}$, which corresponds to the $\mathrm{N}$-terminal band of YipA at $\sim 73 \mathrm{kDa}$. In addition, the YipA C-terminus contains a single predicted protein tyrosine phosphatase (PTP) containing domain (Figure 1B). Thus, it is plausible that YipA is specifically processed for intracellular trafficking to the outer membrane, secretion, or formation of the Tc protein complex and proper activity. Additional studies are necessary to determine the significance of YipA processing events.

Our data show a significant upregulation of the Tc genes in the flea (Figure 2); however, a functional role for the Tc proteins has not been established. Since an infectious dose of greater than 1,000 bacteria is required 
to infect $\sim 50 \%$ of fleas [25], fleas are often fed on a heavily infected blood meal $\left(\sim 1.0 \times 10^{8}-1 \times 10^{9} \mathrm{CFU} / \mathrm{mL}\right)$ to ensure adequate infection. Although these levels of infection are likely seen by fleas feeding on septicemic animals [26,27], fleas may also feed for a shorter duration or on animals with significantly lower numbers of $Y$. pestis in the blood. Under conditions where fewer $Y$. pestis are initially present within the flea, additional $Y$. pestis factors, such as the Tc proteins, may play a more significant role in facilitating survival within the flea and subsequent preventricular blockage and transmission. Thus, we fed fleas on blood containing a low and mid initial dose $\left(\sim 1 \times 10^{7}-1 \times 10^{8}\right)$ of wild-type KIM6+ or KIM6+ 6 itA-yipB. However, even at the lowest initial infectious dose, there were no significant differences between KIM6+ and KIM6+ $y i t A-y i p B$ (Table 1), demonstrating that the Tc proteins are not essential for survival within the flea or for normal proventricular blockage. This is consistent with observations made from fleas infected with a blood meal containing $\sim 1.7 \times 10^{8} \mathrm{CFU} / \mathrm{mL}$ of the KIM6+DyitR mutant [9]. Thus, the $Y$. pestis Tc proteins are not essential for survival within or to produce a normal transmissible infection in the Oriental rat flea $X$. cheopis. However, it is possible that the Tc proteins are important in survival within or transmission from other flea species.

Although we were unable to detect any phenotype in the flea, we were able to localize YitA and YipA to the outer membrane (Figure 6A) and YitA to the surface (Figure 6B) of $Y$. pestis. Thus, they could play a role in infectivity in the mammalian host after transmission. Although the significance of this is yet to be determined, $Y$. pestis from fleas are resistant to phagocytosis and killing by murine and human neutrophils [5,28], and the Tc proteins were implicated in resistance of $Y$. pestis isolated from fleas to phagocytosis by macrophages [9]. Furthermore, the Tc proteins (protein chimeras and full length YipB) were secreted into culture supernatant, Sf9 cells, RAW macrophages, and HeLa cells in a T3SS-dependent manner [18]. However, Y. pseudotuberculosis TcdB protein was detected in both 28 and $37^{\circ} \mathrm{C}$ culture supernatants [16], indicating a T3SS-independent mechanism of Tc protein secretion. Although we saw minimal production of YitA and YipA after prolonged growth at $37^{\circ} \mathrm{C}$, they persisted for several hours after temperature upshift. Therefore, it is plausible that $Y$. pestis Tc proteins produced by $Y$. pestis while in the flea are translocated into mammalian host cells upon transmission, where they act to disrupt the host immune response.

\section{Conclusion}

$Y$. pestis encodes homologues to the $P$. luminescens insecticidal toxins which are highly expressed within the flea vector. However, our data show that $Y$. pestis Tc proteins, unlike P. luminescens toxins [2], are not toxic to fleas and are not essential for survival within the flea midgut or in blockage of the proventriculus. Thus, our data indicate that $Y$. pestis Tc proteins have evolved to limit toxicity to their insect vector. Although the $Y$. pestis Tc proteins may play a yet unidentified important role in survival in the environment, the fact that high levels of YitA and YipA protein are produced by $Y$. pestis while in the flea, and that YitA was identified on the bacterial surface, in addition to other evidence to date $[2,9,16]$, suggests that they are more active against mammalian than insect cells. Thus, $Y$. pestis Tc proteins may have evolved to play a role in subversion of the mammalian immune response, plausibly through resistance to phagocytic cells of the innate immune system or in intracellular survival. Furthermore, our data suggest that since the $Y$. pestis Tc proteins are minimally produced after growth in culture compared to growth in the flea, virulence studies to date using $Y$. pestis grown in broth are inadequate to determine the contribution of Tc proteins, and other proteins specifically upregulated during growth in the flea, in transmission and virulence. Thus, experiments using $Y$. pestis over-producing YitR are underway to determine if the Tc proteins play a role in pathogenicity. Additionally, experiments to determine if $Y$. pestis Tc proteins are secreted or translocated into host neutrophils via the T3SS and their effect on neutrophil phagocytosis and killing are being performed.

\section{Methods}

\section{Bacterial strains, plasmids and culture conditions}

Strains and plasmids used are listed in Table 2. All primers used are listed in Table 3. All experiments were performed under Biosafety Level 2 containment using avirulent $Y$. pestis KIM6+ strains which lack the $\mathrm{pCD} 1$ (Lcr) virulence plasmid and are excluded from CDC Category A Select Agent rules. All transformants were created with approval from the Rocky Mountain Laboratories Institutional Biosafety Committee using approved antibiotic resistance genes. Where indicated, the low-copy plasmid pWKS130::yitR [9] or the high-copy plasmid pCR-XL-TOPO::yitR, created by cloning the PCR-amplified YitR open reading frame flanked by $\sim 300$ bp of upstream and downstream sequence into pCR-XL-TOPO (Life Technologies, Grand Island, NY), was also added to $Y$. pestis to increase YitA and YipA synthesis under broth culture conditions. KIM6+ + yitA-yipB (Figure 1A) was created using the lambda red recombinasemediated knockout procedure described previously [29]. To create both YitA- and YipA- $\beta$-lactamase translational fusions, intermediate pUC19 plasmids which contained the terminal $500 \mathrm{bp}$ of the yitA or yipA open reading frame (minus the stop codon) ligated to the $500 \mathrm{bp}$ immediately downstream of the stop codon were created. Primers used to amplify these regions prior to cloning were flanked with 
Table $2 Y$. pestis strains and plasmids used in this study

\begin{tabular}{|c|c|c|}
\hline Strain or Plasmid & Description $^{a}$ & $\begin{array}{l}\text { Source or } \\
\text { reference }\end{array}$ \\
\hline \multicolumn{3}{|l|}{ Strains } \\
\hline KIM6+ & Tc genes present, pCD1-negative & [33] \\
\hline $\mathrm{KIM} 6+\Delta y i t R$ & Deletion of the regulator YitR & [9] \\
\hline KIM6+ $\Delta$ yitA-yipB & Deletion of the Tc locus from YitA to YipB, YitR still present & This study \\
\hline KIM6+ (pCR-XL-TOPO::yitR) & $\begin{array}{l}\text { KIM6+ transformed with a high-copy plasmid producing } \\
\text { YitR (Kan, Zeo) }\end{array}$ & This study \\
\hline KIM6+ $\triangle$ yitA-yipB (pCR-XL-TOPO::yitR) & $\begin{array}{l}\text { KIM6+ } \Delta \text { yitA-yipB transformed with a high-copy plasmid } \\
\text { producing YitR (Kan, Zeo) }\end{array}$ & This study \\
\hline KIM6+ (pWKS130:yitR) & $\begin{array}{l}\text { KIM6+ transformed with a low-copy plasmid producing } \\
\text { YitR (Kan) }\end{array}$ & This study \\
\hline KIM6+DyitA-yipB (pWKS130::yitR) & $\begin{array}{l}\text { KIM6+ } \Delta y \text { yitA-yipB transformed with a low-copy plasmid } \\
\text { producing YitR (Kan) }\end{array}$ & This study \\
\hline KIM6+ YitA- $\beta$-lactamase & YitA::3-lactamase translational fusion & This study \\
\hline KIM6+ YipA- $\beta$-lactamase & YipA::ß-lactamase translational fusion & This study \\
\hline KIM6+ YitA- $\beta$-lactamase (pCR-XL-TOPO::yitR) & $\begin{array}{l}\text { KIM6+ YitA- } \beta \text {-lactamase transformed with a high-copy plasmid } \\
\text { producing YitR (Kan, Zeo) }\end{array}$ & This study \\
\hline KIM6+ YipA- $\beta$-lactamase (pCR-XL-TOPO::yitR) & $\begin{array}{l}\text { KIM6+ YipA- } \beta \text {-lactamase transformed with a high-copy plasmid } \\
\text { producing YitR (Kan, Zeo) }\end{array}$ & This study \\
\hline KIM6+ (pCR-XL-TOPO::yitR) (pAcGFP1) & $\begin{array}{l}\text { KIM6+ (pCR-XL-TOPO::yitR) transformed with a plasmid producing } \\
\text { GFP (Kan, Zeo, Amp) }\end{array}$ & This study \\
\hline KIM6+DyitA-yipB (pCR-XL-TOPO:yitR) (pAcGFP1) & $\begin{array}{l}\text { KIM6+ } \Delta y i t A-y i p B(p C R-X L-T O P O:-y i t R) \text { transformed with a plasmid } \\
\text { producing GFP (Kan, Zeo, Amp) }\end{array}$ & This study \\
\hline \multicolumn{3}{|l|}{ Plasmids } \\
\hline pCR-XL-TOPO::yitR & $\begin{array}{l}\text { yitR expressed under native promoter from a high-copy plasmid } \\
\text { (Kan, Zeo) }\end{array}$ & This study \\
\hline pWKS130::yitR & yitR expressed under native promoter from a low-copy plasmid (Kan) & [9] \\
\hline pAcGFP1 & Constitutively producing GFP (Amp) & Clontech \\
\hline pUC19 & $\begin{array}{l}\text { Cloning plasmid for creation of YitA- and YipA- } \beta \text {-lactamase fusion } \\
\text { constructs (Amp) }\end{array}$ & [34] \\
\hline pDS132::yitA- $\beta$-lactamase & Suicide plasmid containing YitA- $\beta$-lactamase fusion construct (Cam) & This study \\
\hline pDS132::yipA- $\beta$-lactamase & Suicide plasmid containing YipA- $\beta$-lactamase fusion construct (Cam) & This study \\
\hline pET300/NT-DEST::yitA & Production of YitA with an N-terminal His tag (Amp) & This study \\
\hline pET300/NT-DEST::yipA & Production of YipA with an N-terminal His tag (Amp) & This study \\
\hline
\end{tabular}

$a$ antibiotic resistances where present are noted in parentheses: $K a n=$ Kanamycin, $A m p=$ Ampicillin, $C a m=$ Chloramphenicol, Zeo $=$ Zeocin.

XbaI and XhoI or XhoI/SalI and SphI restriction enzyme sites (Table 3). Following digestion with the appropriate enzymes, the pair of PCR products was cloned into XbaIand SphI-digested pUC19 in a three-way ligation, resulting in recombinant yitA or yipA sequence in which the stop codon was replaced by a 12-nt sequence containing XhoI and Sall restriction sites. The mature domain of TEM- $1 \beta$ lactamase (lacking the $\mathrm{N}$-terminal signal sequence that directs $\beta$-lactamase to the periplasm but including the stop codon) was amplified from pBR322 using primers flanked with XhoI and SalI sites. This fragment was then inserted into both recombinant pUC19 plasmids, resulting in plasmids that contained translational fusions of the YitA or YipA termini with $\beta$-lactamase, linked by the 6 -nt XhoI sequence (introducing the 2 additional amino acids Leu and Glu) and flanked by $500 \mathrm{nt}$ of yitA or yipA downstream sequence following the $\beta$-lactamase stop codon. These constructs were digested from pUC19 using XbaI and SphI, gel purified, and ligated into the suicide vector pDS132 [30]. Recombinant pDS132 plasmids containing yitA- or yipA- $\beta$ lactamase were placed into Escherichia coli S17-1 and transferred from E. coli S17-1 to Y. pestis via conjugation. Transconjugants were selected on Yersinia selective agar [31] with chloramphenicol, and verified by PCR. After overnight growth in brain heart infusion (BHI) broth without selection, transconjugants were placed on BHI agar containing $5 \%$ sucrose to select for allelic exchange mutants [32], which were further screened for chloramphenicol sensitivity and verified by PCR and Western blot analysis using anti-YitA, anti-YipA, and anti- $\beta$-lactamase antibodies (Millipore, Billerica, MA). Y. pestis was grown in BHI broth at $22^{\circ} \mathrm{C}$ overnight from frozen stocks and subcultured into 
Table 3 Primers used for yitR amplification, creation of KIM6+ $\Delta$ yitA-yipB, and creation of YitA and YipA- $\beta$ lactamase fusion proteins

\begin{tabular}{|c|c|}
\hline Primer Designation & Sequence $^{a}$ \\
\hline YitR Forward Primer & $\begin{array}{l}\text { 5'AGTTGAGCTCGTCTGCATTGATTATTT- } \\
\text { GACC-3 }\end{array}$ \\
\hline YitR Reverse Primer & $\begin{array}{l}\text { 5'-AGTTCTAGAGATCGTTGCGTAGCTG- } \\
\text { TGTTGC-3' }\end{array}$ \\
\hline \multirow[t]{2}{*}{ YitA-YipB KO Primer Forward } & $\begin{array}{l}\text { 5'-TGGCATCAATAAACTGGCCTITTCTGT- } \\
\text { TGCAC }\end{array}$ \\
\hline & CAAAAATATGTGTAGGCTGGAGCTGCT-3' \\
\hline \multirow[t]{2}{*}{ YitA-YipB KO Primer Reverse } & $\begin{array}{l}\text { 5'-TTCCCTATTCAAAATAGGGAAGGTGT- } \\
\text { TTAAAA }\end{array}$ \\
\hline & TTAATAAACATATGAATATCCTCCTTA-3' \\
\hline YitA C-terminus Forward & $\begin{array}{l}\text { 5'-ATCTCTAGACCCCAGAACACCCCATG- } \\
\text { TAT-3' }\end{array}$ \\
\hline YitA C-terminus Reverse & $\begin{array}{l}\text { 5'-GATCTCGAGAAGATTAACTCTTAGCTT- } \\
\text { GT-3' }\end{array}$ \\
\hline YitA Downstream Forward & $\begin{array}{l}\text { 5'-ATCCTCGAGGTCGACAAAAACGTTAT- } \\
\text { TACATCCAA-3' }\end{array}$ \\
\hline YitA Downstream Reverse & $\begin{array}{l}\text { 5'-GATGCATGCCTGATTTGACTGATTITI- } \\
\text { CC-3' }\end{array}$ \\
\hline YipA C-terminus Forward & $\begin{array}{l}\text { 5'-ATCTCTAGAAGAGACTTAAAGCATAT- } \\
\text { GGT-3 }\end{array}$ \\
\hline YipA C-terminus Reverse & $\begin{array}{l}\text { 5'-GATCTCGAGTCGATTCTGTTTGCTATA- } \\
\text { TA-3' }\end{array}$ \\
\hline YipA Downstream Forward & $\begin{array}{l}\text { 5'-ATCCTCGAGGTCGACTTAAGAATATT- } \\
\text { AAGGAGCCA-3' }\end{array}$ \\
\hline YipA Downstream Reverse & $\begin{array}{l}\text { 5'-GATGCATGCTGGCCGTTCAGGTTGC- } \\
\text { AGTT-3' }\end{array}$ \\
\hline Mature $\beta$-lactamase Forward & $\begin{array}{l}\text { 5'-ATCCTCGAGCACCCAGAAACGCTGG- } \\
\text { TGA-3 }\end{array}$ \\
\hline Mature $\beta$-lactamase Reverse & $\begin{array}{l}\text { 5'-GATGTCGACTTACCAATGCTTAATCA- } \\
\text { GTGA-3' }\end{array}$ \\
\hline
\end{tabular}

$a$ Underlined sequence denotes restriction enzyme site addition.

fresh $\mathrm{BHI}$ at $22^{\circ} \mathrm{C}$ twice prior to each assay. Where appropriate, kanamycin $(30 \mu \mathrm{g} / \mathrm{mL})$, carbenicillin $(100 \mu \mathrm{g} / \mathrm{mL})$, or chloramphenicol $(10 \mu \mathrm{g} / \mathrm{mL})$ were added to the broth cultures at the indicated final concentration.

\section{YitA and YipA purification and antibody generation}

YitA and YipA were cloned from the Y. pestis KIM Gateway Entry Clone Library created by the Pathogen Functional Genomics Resource Center at the Institute for Genomic Research (TIGR, Rockville, MD). Clones encoding YitA (y0183, TIGR sequence id: 37681/clone id: 141008) and YipA (y0190, sequence id: 37674/clone id: 140911) were used. The yitA and yipA genes were cloned into Champion pET300/NT-DEST vector (Life Technologies) and electroporated into E. coli BL21 (Life Technologies). Production of YitA and YipA after IPTG induction and 4 hours of growth at $37^{\circ} \mathrm{C}$ was verified by SDS-PAGE and by Western blot using anti-6-His antibody (Covance, Princeton, NJ). YitA and YipA proteins were separated by SDS-PAGE and the appropriate-sized bands were excised from the gel, electroeluted and concentrated by centrifugation at $3,200 \mathrm{x} \mathrm{g}$ in centrifugal filters (Amicon Ultra Ultracel $3 \mathrm{~K}$, Millipore). Eluted proteins were further purified by affinity chromatography on nickel-nitrilotriacetic acid (Ni-NTA) resin columns (Qiagen Inc., Valencia, CA). Rabbit polyclonal antiserum was generated against purified YitA (anti-YitA) and YipA (anti-YipA) (Lampire Biological Laboratories, Inc., Pipersville, PA). Non-specific antibodies present in the sera were removed by absorption with $Y$. pestis KIM6+ $6 y i t A-y i p B$ cells [35].

\section{Flea infections and determination of proventricular blockage}

All animals were handled in strict accordance with good animal practice as defined by NIH animal care and use policies and the Animal Welfare Act, USPHS; and all animal work was approved by the Rocky Mountain Laboratories (RML) Animal Care and Use Committee. Fresh mouse blood was obtained from adult RML Swis-Webster mice by cardiac puncture. $X$. cheopis fleas were allowed to feed on an infected blood meal containing $\sim 1 \times 10^{7}$ to $\sim 1 \times 10^{8} \mathrm{CFU} / \mathrm{mL}$ of $Y$. pestis KIM6+ - yitA-yipB or KIM6+ in $5 \mathrm{~mL}$ of fresh heparinized mouse blood. For each infection, 95 female fleas and 55 male fleas that had taken a blood meal were selected. Samples of 20 female fleas were collected immediately after infection (day 0 ) and at 7 and 28 days postinfection and stored at $-80^{\circ} \mathrm{C}$. Throughout the 28 days following infection, fleas were maintained at $22^{\circ} \mathrm{C}$ and fed twice weekly on normal uninfected mice. Immediately after each feeding, fleas were checked by microscopy for blockage of the proventriculus as previously described $[4,36]$. Fleas stored at $-80^{\circ} \mathrm{C}$ were later surface sterilized and individually triturated and plated to determine $Y$. pestis infection rate and mean bacterial load per infected flea as previously described [4].

\section{Western blot analysis of YitA and YipA levels in fleas and liquid media}

2 to 4 weeks after an infectious blood meal containing $2 \times 10^{9} Y$. pestis $/ \mathrm{mL}$, flea midguts were dissected and pooled in lysing matrix H tubes (MP Biomedicals, Solon, $\mathrm{OH}$ ) with $1 \mathrm{~mL}$ Dulbecco's phosphate-buffered saline (DPBS). Tubes containing infected flea midguts were placed in a FastPrep FP120 (Qbiogene, Inc., Carlsbad, CA) homogenizer for $15 \mathrm{~s}$ to triturate midguts and disrupt bacterial aggregates.

Production of YitA and YipA at different temperatures in BHI, heparinized whole sheep blood (Quad Five, Ryegate, MT), or RPMI 1640 (Life Technologies) cell culture media was assessed by subculturing $22^{\circ} \mathrm{C}$ overnight cultures $\left(1: 10\right.$ ratio for $10^{\circ} \mathrm{C}$ culture and 1:20 ratio 
for all other temperatures) into each medium and incubating overnight at $10^{\circ} \mathrm{C}, 22^{\circ} \mathrm{C}, 28^{\circ} \mathrm{C}$ or $37^{\circ} \mathrm{C}$. All cultures had an OD $600 \mathrm{~nm}$ between 1.2 and 2.0 prior to processing.

Persistence of YitA and YipA following transfer of $Y$. pestis grown at $22^{\circ} \mathrm{C}$ to $37^{\circ} \mathrm{C}$ was assessed by taking $100 \mathrm{~mL}$ overnight BHI cultures of KIM6+ (pCR-XLTOPO::yitR) or KIM6+_yitA-yipB (pCR-XL-TOPO::yitR) grown at $22^{\circ} \mathrm{C}$ and transferring them to $37^{\circ} \mathrm{C}$. A $100 \mathrm{~mL}$ culture of KIM6+ (pCR-XL-TOPO::yitR) was kept at $22^{\circ} \mathrm{C}$ as a positive control. Samples were taken from the cultures 1 to $30 \mathrm{~h}$ after transfer.

For Western blot analysis, all bacteria were pelleted, washed, resuspended in DPBS and quantified by PetroffHausser direct counts. Samples were normalized to equivalent cell numbers and the lysates of approximately $3 \times 10^{7}$ bacteria (grown in broth or isolated from fleas) were separated by SDS-PAGE in lanes of $4-15 \%$ precast polyacrylamide gels (Criterion TGX, Bio-rad, Hercules, CA). Samples were then transferred to $0.2 \mu \mathrm{m}$ nitrocellulose for Western blot analysis. YitA and YipA were detected using anti-YitA or anti-YipA serum. Mouse antiserum against the constitutively expressed $Y$. pestis outer membrane protein Ail [37] was used for a sample loading control. Goat anti-rabbit IgG or goat antimouse IgG antibodies conjugated to alkaline phosphatase (Life Technologies) and BCIP/NBT-Blue liquid substrate (Sigma-Aldrich, St. Louis, MO) were used to visualize protein bands.

\section{Fractionation of $Y$. pestis}

$Y$. pestis was grown overnight in $\mathrm{BHI}$ at $22^{\circ} \mathrm{C}$ and subcultured into $500 \mathrm{~mL}$ of fresh $\mathrm{BHI}$ at a 1:100 ratio. Cultures were grown overnight with aeration at $22^{\circ} \mathrm{C}$. Bacteria were pelleted, washed, and the cytoplasmic, periplasmic, cytosolic membrane, and outer membrane fractions were collected using a previously described protocol [38]. The total protein concentration of the fractions was determined (Qubit Fluorometer Protein Assay Kit, Life Technologies) and normalized to $1.0 \mathrm{mg} / \mathrm{mL}$ of total protein. For Western blot analysis, $30 \mu \mathrm{g}$ of each fraction was loaded per well.

\section{Immunofluorescence microscopy}

$Y$. pestis KIM6+ (pCR-XL-TOPO::yitR) (pAcGFP1), or KIM6+ $y$ itA-yipB (pCR-XL-TOPO::yitR), (pAcGFP1) as a negative control, were grown overnight in $\mathrm{BHI}$ at $22^{\circ} \mathrm{C}$. Bacteria were pelleted and washed two times and resuspended in PBS. Bacteria were added to glass coverslips in 24-well microtiter plates and centrifuged at $3,000 \mathrm{x} \mathrm{g}$ for $10 \mathrm{~min}$. Bacteria were fixed in $4 \%$ paraformaldehyde for $15 \mathrm{~min}$ at $37^{\circ} \mathrm{C}$ and washed. Bacteria were incubated with anti-YitA or anti-YipA rabbit serum for $30 \mathrm{~min}$ at $37^{\circ} \mathrm{C}$, washed, stained with Alexa Fluor 568 goat antirabbit IgG (Life Technologies), and imaged by fluorescence microscopy. Pictures were taken using a Photometrics CoolSnap HQ black and white camera and images were artificially colored and combined using MetaMorph software version 7.5.6.0 (Molecular Devices, Sunnyvale, CA).

\section{Competing interests}

The author(s) declare that they have no competing interests.

\section{Authors' contributions}

$J \mathrm{LS}$ and $\mathrm{BJH}$ wrote the manuscript. JLS, COJ, DLL, CMC and BJH conceived of and participated in the design of the study. JLS, COJ, and BJH performed the experiments. COJ created $Y$. pestis $\mathrm{KIM} 6+\Delta y$ itR. DLL created $Y$. pestis $\mathrm{KIM6}+$ $\triangle$ yitA-yipB. SIM, CMC, and BJH provided materials and reagents. All authors read and approved the final manuscript.

\section{Acknowledgements}

We thank Madeline Fasen, and the RML visual and medical arts staff for their assistance. We thank Chris Bosio, Jeffrey Shannon, Iman Chouikha, Sophia Dudte, and Aaron Hasenkrug for critical review of the manuscript. This research was supported by the Intramural Research Program of the NIAID, $\mathrm{NIH}$ and by the NIH Grant R21 Al067444.

\section{Author details}

${ }^{1}$ Laboratory of Zoonotic Pathogens, Rocky Mountain Laboratories, NIAID, NIH, Hamilton, MT 59840, USA. ²Department of Microbiology, University of Washington, Seattle, WA 98195, USA. ${ }^{3}$ Department of Genome Sciences, Immunology, and Medicine, University of Washington, Seattle, WA 98195 USA.

Received: 15 October 2012 Accepted: 12 December 2012 Published: 18 December 2012

\section{References}

1. Erickson DL, Jarrett CO, Wren BW, Hinnebusch BJ: Serotype differences and lack of biofilm formation characterize Yersinia pseudotuberculosis infection of the Xenopsylla cheopis flea vector of Yersinia pestis. J Bacteriol 2006, 188(3):1113-1119.

2. Erickson DL, Waterfield NR, Vadyvaloo V, Long D, Fischer ER, ffrench-Constant RH, Hinnebusch BJ: Acute oral toxicity of Yersinia pseudotuberculosis to fleas: implications for the evolution of vector-borne transmission of plague. Cell Microbiol 2007, 9:2658-2666.

3. Achtman M, Zurth K, Morelli G, Torrea G, Guiyoule A, Carniel E: Yersinia pestis, the cause of plague, is a recently emerged clone of Yersinia pseudotuberculosis. Proc Natl Acad Sci USA 1999, 96(24):14043-14048.

4. Hinnebusch BJ, Perry RD, Schwan TG: Role of the Yersinia pestis hemin storage (hms) locus in the transmission of plague by fleas. Science 1996, 273(5273):367-370

5. Jarrett CO, Deak E, Isherwood KE, Oyston PC, Fischer ER, Whitney AR, Kobayashi SD, DeLeo FR, Hinnebusch BJ: Transmission of Yersinia pestis from an infectious biofilm in the flea vector. J Inf Dis 2004, 190:783-792.

6. Darby C, Ananth SL, Tan L, Hinnebusch BJ: Identification of gmhA, a Yersinia pestis gene required for flea blockage, by using a Caenorhabditis elegans biofilm system. Infect Immun 2005, 73(11):7236-7242.

7. Sun YC, Koumoutsi A, Jarrett C, Lawrence K, Gherardini FC, Darby C, Hinnebusch BJ: Differential control of Yersinia pestis biofilm formation in vitro and in the flea vector by two c-di-GMP diguanylate cyclases. PLoS One 2011, 6(4):e19267.

8. Hinnebusch BJ, Rudolph AE, Cherepanov P, Dixon JE, Schwan TG, Forsberg $\AA$ : Role of Yersinia murine toxin in survival of Yersinia pestis in the midgut of the flea vector. Science 2002, 296:733-735.

9. Vadyvaloo V, Jarrett C, Sturdevant DE, Sebbane F, Hinnebusch BJ: Transit through the flea vector induces a pretransmission innate immunity resistance phenotype in Yersinia pestis. PLoS Pathogens 2010, 6:e10000783.

10. Bowen D, Rocheleau TA, Blackburn M, Andreev O, Golubeva E, Bhartia R, ffrench-Constant $\mathrm{RH}$ : Insecticidal toxins from the bacterium Photorhabdus luminescens. Science 1998, 280(5372):2129-2132. 
11. Waterfield NR, Bowen DJ, Fetherston JD, Perry RD, ffrench-Constant RH: The tc genes of Photorhabdus: a growing family. Trends Microbiol 2001, 9(4):185-191.

12. Fuchs TM, Bresolin G, Marcinowski L, Schachtner J, Scherer S: Insecticidal genes of Yersinia spp.: taxonomical distribution, contribution to toxicity towards Manduca sexta and Galleria mellonella, and evolution. BMC Microbiol 2008, 8:214.

13. Munch A, Stingl L, Jung K, Heermann R: Photorhabdus luminescens genes induced upon insect infection. BMC Genomics 2008, 9:229.

14. Waterfield NR, Dowling A, Sharma S, Daborn PJ, Potter U, ffrench-Constant RH: Oral toxicity of Photorhabdus luminescens W14 toxin complexes in Escherichia coli. Appl Environ Microbiol 2001, 67:5017-5024

15. Waterfield NR, Hares M, Yang G, Dowling A, ffrench-Constant RH: Potentiation and cellular phenotypes of the insecticidal toxin complexes of Photorhabdus bacteria. Cell Microbiol 2005, 7(3):373-382.

16. Hares MC, Hinchliffe SJ, Strong PC, Eleftherianos I, Dowling AJ, ffrench-Constant RH, Waterfield NR: The Yersinia pseudotuberculosis and Yersinia pestis toxin complex is active against cultured mammalian cells. Microbiology 2008, 154(Pt 11):3503-3517.

17. Lang AE, Schmidt G, Schlosser A, Hey TD, Larrinua IM, Sheets JJ, Mannherz HG, Aktories K: Photorhabdus luminescens toxins ADP-ribosylate actin and RhoA to force actin clustering. Science 2010, 327(5969):1139-1142.

18. Gendlina I, Held KG, Bartra SS, Gallis BM, Doneanu CE, Goodlett DR, Plano GV, Collins CM: Identification and type III-dependent secretion of the Yersinia pestis insecticidal-like proteins. Mol Microbiol 2007, 64(5):1214-1227

19. Motin VL, Georgescu AM, Fitch JP, Gu PP, Nelson DO, Mabery SL, Garnham JB, Sokhansanj BA, Ott LL, Coleman MA, et al: Temporal global changes in gene expression during temperature transition in Yersinia pestis. J Bacterio/ 2004, 186(18):6298-6305.

20. Sebbane F, Lemaitre N, Sturdevant DE, Rebeil R, Virtaneva K, Porcella SF, Hinnebusch BJ: Adaptive response of Yersinia pestis to extracellular effectors of innate immunity during bubonic plague. Proc Natl Acad Sci USA 2006, 103:11766-11771.

21. Pinheiro VB, Ellar DJ: Expression and insecticidal activity of Yersinia pseudotuberculosis and Photorhabdus luminescens toxin complex proteins. Cell Microbio/ 2007, 9:2372-2380.

22. Bresolin G, Morgan JA, Ilgen D, Scherer S, Fuchs TM: Low temperatureinduced insecticidal activity of Yersinia enterocolitica. Mol Microbiol 2006, 59(2):503-512.

23. Fukuto HS, Svetlanov A, Palmer LE, Karzai AW, Bliska JB: Global gene expression profiling of Yersinia pestis replicating inside macrophages reveals the roles of a putative stress-induced operon in regulating type III secretion and intracellular cell division. Infect Immun 2010, 78(9):3700-3715.

24. Hinnebusch BJ, Sebbane F, Vadyvaloo V: Transcriptional profiling of the Yersinia pestis life cycle. In Yersinia: systems biology and control. Edited by Carniel E, Hinnebusch BJ. Norfolk, UK: Caister Academic Press; 2012:1-18.

25. Lorange EA, Race BL, Sebbane F, Hinnebusch BJ: Poor vector competence of fleas and the evolution of hypervirulence in Yersinia pestis. $J$ Inf Dis 2005, 191:1907-1912.

26. Comer JE, Sturdevant DE, Carmody AB, Virtaneva K, Gardner D, Long D, Rosenke R, Porcella SF, Hinnebusch BJ: Transcriptomic and innate immune responses to Yersinia pestis in the lymph node during bubonic plague. Infect Immun 2010, 78:5086-5098.

27. Sebbane F, Jarrett CO, Gardner D, Long D, Hinnebusch BJ: Role of the Yersinia pestis plasminogen activator in the incidence of distinct septicemic and bubonic forms of flea-borne plague. Proc Natl Acad Sci USA 2006, 103:5526-5530.

28. Spinner JL, Hinnebusch BJ: The life stage of Yersinia pestis in the flea vector confers increased resistance to phagocytosis and killing by murine polymorphonuclear leukocytes. Adv Exp Med Biol 2012, 954:159-163.

29. Datsenko KA, Wanner BL: One-step inactivation of chromosomal genes in Escherichia coli K-12 using PCR products. Proc Natl Acad Sci USA 2000, 97(12):6640-6645.

30. Philippe N, Alcaraz JP, Coursange E, Geiselmann J, Schneider D: Improvement of pCVD442, a suicide plasmid for gene allele exchange in bacteria. Plasmid 2004, 51(3):246-255.
31. Schiemann DA: Synthesis of a selective agar medium for Yersinia enterocolitica. Can J Microbiol 1979, 25(11):1298-1304.

32. Donnenberg MS, Kaper JB: Construction of an eae deletion mutant of enteropathogenic Escherichia coli by using a positive-selection suicide vector. Infect Immun 1991, 59(12):4310-4317.

33. Une T, Brubaker RR: In vivo comparison of avirulent Vwa- and Pgm- or Pstr phenotypes of yersiniae. Infect Immun 1984, 43(3):895-900.

34. Yanisch-Perron C, Vieira J, Messing J: Improved M13 phage cloning vectors and host strains: nucleotide sequences of the M13mp18 and pUC19 vectors. Gene 1985, 33(1):103-119.

35. Wendelboe HG, Bisgaard K: Contaminating antibodies and cross-reactivity. In Immunohistochemical (IHC) staining methods. 5th edition. Edited by Kumar GL, Rudbeck L. Carpinteria, CA: Dako; 2009.

36. Hinnebusch BJ, Fischer ER, Schwan TG: Evaluation of the role of the Yersinia pestis plasminogen activator and other plasmid-encoded factors in temperature-dependent blockage of the flea. J Inf Dis 1998, 178(5):1406-1415.

37. Yamashita S, Lukacik P, Barnard TJ, Noinaj N, Felek S, Tsang TM, Krukonis ES, Hinnebusch BJ, Buchanan SK: Structural insights into Ail-mediated adhesion in Yersinia pestis. Structure 2011, 19(11):1672-1682.

38. Thein M, Sauer G, Paramasivam N, Grin I, Linke D: Efficient subfractionation of gram-negative bacteria for proteomics studies. J Proteome Res 2010 9(12):6135-6147.

doi:10.1186/1471-2180-12-296

Cite this article as: Spinner et al: Yersinia pestis insecticidal-like toxin complex (Tc) family proteins: characterization of expression, subcellular localization, and potential role in infection of the flea vector. BMC Microbiology 2012 12:296.

\section{Submit your next manuscript to BioMed Central and take full advantage of:}

- Convenient online submission

- Thorough peer review

- No space constraints or color figure charges

- Immediate publication on acceptance

- Inclusion in PubMed, CAS, Scopus and Google Scholar

- Research which is freely available for redistribution 